\title{
VARIAÇÕES FLORÍSTICO-ESTRUTURAIS DA COMUNIDADE ARBÓREA ASSOCIADAS À DISTÂNCIA DA BORDA EM UM FRAGMENTO FLORESTAL NO PLANALTO SUL-CATARINENSE
}

\author{
Francieli Pscheidt ${ }^{1}$, Carla Carolina Chini Rech ${ }^{1}$, Francieli de Fátima Missio ${ }^{1}$, Marco Antônio Bento ${ }^{2}$, \\ Fernando Buzzi Junior ${ }^{2}$, Roni Djeison Ansolin ${ }^{2}$, Marcelo Bonazza ${ }^{1}$, Manoela Drews de Aguiar ${ }^{3}$, \\ Ana Carolina da Silva ${ }^{4}$, Pedro Higuchi ${ }^{4}$
${ }^{1}$ Universidade do Estado de Santa Catarina, Programa de Pós-Graduação em Engenharia Florestal, Lages, Santa Catarina, Brasil - francieli_pscheidt@hotmail.com; karol_chini@hotmail.com; franmissio@yahoo.com.br; marcelo.bonazza@hotmail.com
${ }^{2}$ Universidade do Estado de Santa Catarina, Curso de Engenharia Florestal, Lajes, Santa Catarina, Brasil - marco_a bento@hotmail.com; buzzifjr@hotmail.com; roni_ansolin@hotmail.com
${ }^{3}$ Universidade Regional de Blumenau, Blumenau, Santa Catarina, Brasil - manoo_aguiar@ hotmail.com
${ }^{4}$ Universidade do Estado de Santa Catarina, Departamento Engenharia Florestal, Lajes, Santa Catarina, Brasil - carol_sil4@yahoo.com.br; higuchip@gmail.com

Recebido para publicação: 02/11/2013 - Aceito para publicação: 08/08/2014

\begin{abstract}
Resumo
Com o propósito de comparar a organização florístico-estrutural de uma comunidade de espécies arbóreas nos setores de borda e interior em um fragmento de Floresta Ombrófila Mista localizado no município de Lages, SC, foi realizado um levantamento vegetacional em cinco transeções de 100 × 20 m, subdivididas em parcelas de $10 \times 20 \mathrm{~m}$, perpendiculares à borda do fragmento. Todas as árvores com circunferência na altura do peito (CAP) $\geq 15,7 \mathrm{~cm}$ foram mensuradas (CAP e altura) e determinadas. Os dados foram analisados por meio do índice de valor de importância (IVI), da análise de correspondência retificada (DCA), da análise de variância multivariada não paramétrica (NPMANOVA), dos valores médios de abundância, área basal, altura, riqueza, diversidade e equabilidade e da frequência de indivíduos em classes de diâmetro e altura. Apesar de tanto o setor borda quando o interior apresentarem Araucaria angustifolia (Bertol.) Kuntze e Lithraea brasilisiensis Marchand entre as espécies de maior IVI, houve substituição nas demais posições de IVI, que refletiu na variação da organização da comunidade entre os setores $(p=0,022)$, também observada na DCA. Em relação aos valores médios das demais variáveis mensuradas, não foram encontradas diferenças. Conclui-se que a borda representara uma importante fonte de heterogeneidade florístico-estrutural no fragmento estudado.

Palavras-chave: Fragmentação; floresta com araucárias; espécies arbóreas.
\end{abstract}

\begin{abstract}
Floristic-structural variations of a tree community associated to edge distance in a forest patch in "Planalto Sul Catarinense" region. In order to compare the floristic-structural organization of a tree species community in an Araucaria Forest patch, in the municipality of Lages, SC, a phytossociological survey was conducted in five $100 \times 20 \mathrm{~m}$ transections, subdivided into $10 \times 20 \mathrm{~m}$ plots, perpendicular to the patch edges. It were measured and determined all trees with circumference at breast height $(\mathrm{dbh}) \geq 15,7$ (cbh and height). The data were analyzed by the importance value index (IVI), Detrended Correspondence Analysis (DCA); non-parametric multivariate analyze of variance (NPMANOVA); mean values of abundance, basal area, tree height, richness, diversity and evenness; and frequency of individuals within diameter and height classes. Although both sectors had Araucaria angustifolia (Bertol.) Kuntze and Lithraea brasiliensis Marchand among the species with the highest IVI values, there was a turnover in other IVI positions, which reflected in the variation of tree species organization among sectors $(p=0.022)$, also observed in DCA. Related to community structure (mean values of abundance and tree height and individuals frequency in size classes), no differences were detected. We conclude that edges represented an important source of floristic-structural heterogeneity in the studied patch.
\end{abstract}

Keywords: Fragmentation; araucaria forest; tree species.

FLORESTA, Curitiba, PR, v. 45, n. 2, p. 421 - 430, abr. / jun. 2015.

Pscheidt, F. et al.

ISSN eletrônico 1982-4688 / ISSN impresso 0015-3826 


\section{INTRODUÇÃO}

Em decorrência do avanço das ações humanas na maior parte dos remanescentes florestais, a Mata Atlântica se encontra atualmente fragmentada, com áreas geralmente perturbadas e pouco protegidas, apresentando alterações na estrutura das comunidades e populações de espécies arbóreas (VIANA; PINHEIRO, 1998). Esse cenário também é observado na Floresta Ombrófila Mista (FOM), que representa uma das principais fitofisionomias da Mata Atlântica na região Sul do Brasil, cujos remanescentes representam apenas $12,6 \%$ de sua área de cobertura original (RIBEIRO et al., 2009). No estado de Santa Catarina, em função da conversão do solo para atividades agropecuárias e silviculturais, a FOM encontra-se atualmente distribuída na paisagem na forma de mosaicos de vegetação em diferentes estágios sucessionais e de perturbação (BAPTISTA; RUDEL, 2006).

A configuração de fragmentos implica a formação de bordas florestais, onde a área alterada em contato com a vegetação original influencia a dinâmica da floresta em função das diferentes respostas das espécies (HIGUCHI et al., 2008). Essas alterações nas partes externas dos fragmentos florestais são chamadas "efeitos de borda" e causam impacto sobre os organismos que habitam esses ambientes (MURCIA, 1995), pois podem alterar a composição ou a abundância relativa de espécies (FORMAN; GRODON, 1986). Mudança no microclima e na estrutura física dos fragmentos, redução da heterogeneidade ambiental e extinções locais são algumas consequências desse processo, que segue da direção da borda para o interior do fragmento (MURCIA, 1995; YOUNG; MITCHELL, 1994).

Apesar de alguns estudos florísticos e fitossociológicos terem sido realizados na FOM em Santa Catarina (FORMENTO et al., 2004; ESKUCHE, 2007; KLAUBERG et al., 2010; NASCIMENTO et al., 2011; HIGUCHI et al., 2012; SILVA et al., 2012; HIGUCHI et al., 2013), existem poucos estudos que investigam o efeito de borda nessas formações (FONTOURA et al., 2006). Assim, o presente trabalho objetivou analisar as variações florístico-estruturais e de diversidade da comunidade de espécies arbóreas entre os setores de borda e interior, em um fragmento de FOM, no Planalto Sul-Catarinense, com o propósito de caracterizar a influência do efeito de borda sobre o componente arbóreo no fragmento estudado.

\section{MATERIAL E MÉTODOS}

O estudo foi realizado em um fragmento florestal no município de Lages, Santa Catarina, localizado na latitude $27^{\circ} 51^{\prime} 13$ ' S e longitude $50^{\circ} 09^{\prime} 47^{\prime \prime} \mathrm{O}$, com altitude em torno de $1.080 \mathrm{~m}$. A região é classificada, segundo Köppen, como clima $\mathrm{Cfb}$, temperado constantemente úmido, sem estação seca. A precipitação anual média é de $1.479,48 \mathrm{~mm}$, bem distribuídas no ano, e a temperatura anual média é de $16{ }^{\circ} \mathrm{C}$. O município está inserido nas bacias hidrográficas dos rios Canoas e Pelotas, formadores do rio Uruguai, com relevo predominantemente suave-ondulado a ondulado, sendo a vegetação regional classificada como Floresta Ombrófila Mista Montana e Estepes (INSTITUTO BRASILEIRO DE GEOGRAFIA E ESTATÍSTICA (IBGE), 2012).

Para análise da vegetação, foram estabelecidas cinco transeções perpendiculares à borda do fragmento florestal, com extensão de $100 \mathrm{~m}$ para o interior da floresta e largura de $20 \mathrm{~m}$, totalizando $2.000 \mathrm{~m}^{2}$ por transeção e 1 ha de área total amostrada. $\mathrm{O}$ fragmento florestal faz parte da reserva legal de uma propriedade rural e encontra-se protegido de distúrbios de origem antrópica. Com o propósito de amostrar a heterogeneidade existente, as transeções foram alocadas distanciadas, pelo menos, $100 \mathrm{~m}$ entre si. Cada uma das transeções foi subdividida em dez subparcelas de 10 x $20 \mathrm{~m}$. Foram consideradas como setor "borda" as cinco primeiras parcelas $(0-50 \mathrm{~m})$ de cada transecção e como setor "interior" as cinco últimas parcelas $(50-100 \mathrm{~m})$. Em cada parcela, todos os indivíduos arbóreos vivos que apresentaram circunferência à altura do peito (CAP) (medida a $1,30 \mathrm{~m}$ do solo) igual ou superior a $15,7 \mathrm{~cm}$ foram identificados e marcados com plaquetas de alumínio. Em cada um desses indivíduos, foi medido o CAP, com fita métrica, e estimada a altura. As espécies foram classificadas nas famílias de acordo com o sistema APG III (ANGIOSPERM PHYLOGENY GROUP, 2009).

A suficiência amostral foi determinada analisando-se a curva de acumulação de espécies rarefeita, com riqueza estimada por meio de 1.000 permutações. A estrutura horizontal foi descrita, para os setores "borda" e "interior", a partir do cálculo do índice de valor de importância (IVI) para cada espécie, determinado, segundo Mueller-Dombois e Ellemberg (1974), por meio da média da densidade relativa, frequência relativa e dominância relativa. Os setores foram comparados por meio das 10 espécies com maior IVI. A fim de verificar a organização estrutural da comunidade arbórea, as parcelas foram 
ordenadas por meio de uma análise de correspondência retificada (DCA), utilizando-se dados de abundância, com transformação logarítmica log $(x+1)$. Como sugerido por Anderson (2001) para a análise de dados multivariados de comunidades ecológicas, a existência de diferenças significativas entre os setores em relação à composição florístico-estrutural foi verificada por uma análise de variância não paramétrica multivariada (NPMANOVA). A comparação da abundância (densidade), área basal, altura média, riqueza, diversidade (Índice de Shannon) e equabilidade (Índice de Pielou) entre os setores borda e interior foi realizada por meio do teste $t$, após a verificação da natureza paramétrica dos dados, usando-se o teste de Shapiro-Wilk. Para a análise da estrutura diamétrica e hipsométrica da comunidade dos diferentes setores, foram utilizados histogramas de frequência, com intervalos de classes (IC) determinados a partir da fórmula de Spiegel (FELFILI; RESENDE, 2003): IC $=\mathrm{A} / \mathrm{nc}$, em que A é a amplitude e nc o número de classes, sendo $\mathrm{nc}=1+3,3 \log (\mathrm{n})$, em que $\mathrm{n}$ é o número de indivíduos. As fórmulas foram aplicadas aos dados da comunidade para a definição do número de classes e das amplitudes para diâmetro e altura. As análises dos dados foram realizadas utilizando-se o programa $\mathrm{R}$ (R DEVELOPMENT CORE TEAM, 2013), junto com o pacote Vegan (OKSANEN et al., 2013).

\section{RESULTADOS E DISCUSSÃO}

Foram amostrados 1.514 indivíduos, pertencentes a 67 espécies, 47 gêneros e 30 famílias (Tabela 1). Myrtaceae se destacou como a família de maior riqueza (15 espécies), tanto no setor "borda" (11) como no "interior" (12). Esse resultado confirma o padrão da FOM da região, em que Myrtaceae tem se destacado com maior número de espécies (KLAUBERG et al., 2010; NASCIMENTO et al., 2011; HIGUCHI et al., 2012; SILVA et al., 2012; HIGUCHI et al., 2013). Cinco espécimes não foram identificados por estarem sem folhas. A curva de acumulação (Figura 1) indicou que a suficiência amostral foi atingida, pois, com um aumento de $10 \%$ na área amostral, houve um aumento de apenas $2,11 \%$ no número de espécies. Atinge-se a suficiência quando a curva tende à estabilidade e a adição de novas unidades amostrais não altera significativamente o número de espécies observadas, sendo sugerido que um aumento de 10\% na área amostral resulte em um aumento de até $5 \%$ em novas espécies (MATTEUCCI; COLMA, 1982; KERSTEN; GALVÃO, 2011).

Tabela 1. Espécies amostradas nos setores "borda" e "interior" do fragmento estudado de Floresta Ombrófila Mista em Lages, SC.

Table 1. Tree species sampled in 'edge' and 'interior' sectors in the studied Araucaria Forest patch, in Lages, SC.

\begin{tabular}{|c|c|c|c|}
\hline Família/Espécie & Borda & Interior & nc \\
\hline \multicolumn{4}{|l|}{ ANACARDIACEAE } \\
\hline Lithraea brasiliensis Marchand & 50 & 47 & 7678 \\
\hline Schinus lentiscifolius Marchand & 2 & - & - \\
\hline Schinus terebinthifolius Raddi & 5 & 1 & 7665 \\
\hline \multicolumn{4}{|l|}{ ANNONACEAE } \\
\hline Annona rugulosa (Schltdl.) H.Rainer & 3 & 7 & - \\
\hline \multicolumn{4}{|l|}{ AQUIFOLIACEAE } \\
\hline Ilex dumosa Reissek & 6 & 3 & 7671 \\
\hline Ilex microdonta Reissek & 7 & 5 & 7674 \\
\hline Ilex theezans Mart. ex Reissek & 12 & 11 & 7664 \\
\hline \multicolumn{4}{|l|}{ ARAUCARIACEAE } \\
\hline Araucaria angustifolia (Bertol.) Kuntze & 83 & 69 & 7719 \\
\hline \multicolumn{4}{|l|}{ ASTERACEAE } \\
\hline Dasyphyllum brasiliense (Spreng.) Cabrera & - & 1 & 7657 \\
\hline Gochnatia polymorpha (Less.) Cabrera & 6 & 2 & 7682 \\
\hline Vernonanthura discolor (Spreng.) H.Rob. & 17 & 15 & 7690 \\
\hline \multicolumn{4}{|l|}{ BIGNONIACEAE } \\
\hline Jacaranda puberula Cham. & 19 & 61 & 7715 \\
\hline \multicolumn{4}{|l|}{ CELASTRACEAE } \\
\hline Maytenus boaria Molina & 3 & - & - \\
\hline Maytenus dasyclada Mart. & 6 & 7 & 7655 \\
\hline
\end{tabular}

FLORESTA, Curitiba, PR, v. 45, n. 2, p. 421 - 430, abr. / jun. 2015.

Pscheidt, F. et al.

ISSN eletrônico 1982-4688 / ISSN impresso 0015-3826

DOI: $10.5380 /$ rf.v45i2.34180 


\begin{tabular}{|c|c|c|c|}
\hline \multicolumn{4}{|l|}{ CLETHRACEAE } \\
\hline Clethra scabra Pers. & 14 & 14 & 7667 \\
\hline \multicolumn{4}{|l|}{ CUNONIACEAE } \\
\hline Lamanonia ternate Vell. & 5 & 27 & 7650 \\
\hline \multicolumn{4}{|l|}{ DICKSONIACEAE } \\
\hline Dicksonia sellowiana Hook. & 18 & 40 & 7717 \\
\hline \multicolumn{4}{|l|}{ ERYTHROXYLACEAE } \\
\hline Erythroxylum deciduum A.St.-Hil. & 3 & 1 & 7683 \\
\hline \multicolumn{4}{|l|}{ EUPHORBIACEAE } \\
\hline Sapium glandulosum (L.) Morong & 6 & 11 & 7677 \\
\hline Sebastiania brasiliensis Spreng. & - & 11 & 7670 \\
\hline Sebastiania commersoniana (Baill.) L.B.Sm. \& Downs & 1 & 15 & 7684 \\
\hline \multicolumn{4}{|l|}{ FABACEAE } \\
\hline Dalbergia frutescens (Vell.) Britton & 5 & 6 & 7689 \\
\hline Inga lentiscifolia Benth. & - & 1 & 7688 \\
\hline Machaerium paraguariense Hassl. & - & 3 & 7680 \\
\hline \multicolumn{4}{|l|}{ LAURACEAE } \\
\hline Cinnamoтит атоепит (Nees \& Mart.) Kosterm. & 2 & 8 & 7691 \\
\hline Ocotea puberula (Rich.) Nees & 1 & - & 7681 \\
\hline Ocotea pulchella (Nees \& Mart.) Mez & 14 & 24 & 7666 \\
\hline \multicolumn{4}{|l|}{ LOGANIACEAE } \\
\hline Strychnos brasiliensis (Spreng.) Mart. & 1 & 2 & 7663 \\
\hline \multicolumn{4}{|l|}{ MYRTACEAE } \\
\hline Acca sellowiana (O.Berg) Burret & 3 & 4 & 7676 \\
\hline Blepharocalyx salicifolius (Kunth) O.Berg & 29 & 11 & 7706 \\
\hline Calyptranthes concinna DC. & 57 & 51 & 7700 \\
\hline Campomanesia xanthocarpa O.Berg & - & 1 & 7698 \\
\hline Eugenia pluriflora DC. & 11 & 4 & 7701 \\
\hline Eugenia pyriformis Cambess. & - & 2 & - \\
\hline Eugenia uruguayensis Cambess. & - & 2 & 7716 \\
\hline Myrceugenia euosma (O.Berg) D.Legrand & 1 & - & 7704 \\
\hline Myrceugenia glaucescens (Cambess.) D.Legrand \& Kausel & 1 & - & 7707 \\
\hline Myrceugenia oxysepala (Burret) D.Legrand \& Kausel & 1 & 4 & 7694 \\
\hline Myrcia guianensis (Aubl.) DC. & 28 & 21 & 7702 \\
\hline Myrcia hartwegiana (O.Berg) Kiaersk. & 2 & - & 7705 \\
\hline Myrcia laruotteana Cambess. & 7 & 7 & 7699 \\
\hline Myrcia palustris DC. & 49 & 42 & 7703 \\
\hline Myrrhinium atropurpureum Schott & - & 1 & 7659 \\
\hline NÃO IDENTIFICADA & 2 & 3 & - \\
\hline \multicolumn{4}{|l|}{ PODOCARPACEAE } \\
\hline Podocarpus lambertii Klotzsch ex Endl. & 52 & 32 & 7673 \\
\hline \multicolumn{4}{|l|}{ PRIMULACEAE } \\
\hline Myrsine coriacea (Sw.) R.Br. ex Roem. \& Schult. & 4 & 1 & 7654 \\
\hline Myrsine lorentziana (Mez) Arechav. & 49 & 23 & 7653 \\
\hline Myrsine umbellata Mart. & 9 & 9 & 7652 \\
\hline \multicolumn{4}{|l|}{ PROTEACEAE } \\
\hline Roupala montana Aubl. & - & 2 & 7651 \\
\hline \multicolumn{4}{|l|}{ QUILLAJACEAE } \\
\hline Quillaja brasiliensis (A.St.-Hil. \& Tul.) Mart. & 1 & - & 7675 \\
\hline \multicolumn{4}{|l|}{ RHAMNACEAE } \\
\hline Scutia buxifolia Reissek & 15 & 5 & 7685 \\
\hline \multicolumn{4}{|l|}{ ROSACEAE } \\
\hline Prunus myrtifolia (L.) Urb. & 5 & 21 & 7658 \\
\hline RUTACEAE & & & \\
\hline
\end{tabular}

FLORESTA, Curitiba, PR, v. 45, n. 2, p. 421 - 430, abr. / jun. 2015. 


\begin{tabular}{|c|c|c|c|}
\hline Zanthoxylum kleinii (R.S.Cowan) P.G.Waterman & 20 & 8 & 7668 \\
\hline Zanthoxylum rhoifolium Lam. & 2 & 13 & 7669 \\
\hline \multicolumn{4}{|l|}{ SALICACEAE } \\
\hline Banara tomentosa Clos & - & 3 & 7686 \\
\hline Casearia decandra Jacq. & 47 & 31 & 7692 \\
\hline Casearia obliqua Spreng. & 1 & 9 & 7714 \\
\hline Xylosma ciliatifolia (Clos) Eichler & 2 & 2 & 7687 \\
\hline Xylosma tweediana (Clos) Eichler & 1 & - & - \\
\hline \multicolumn{4}{|l|}{ SAPINDACEAE } \\
\hline Allophylus edulis (A.St.-Hil., Cambess. \& A. Juss.) Radlk. & 11 & 11 & 7679 \\
\hline Matayba elaeagnoides Radlk. & - & 10 & 7697 \\
\hline \multicolumn{4}{|l|}{ SOLANACEAE } \\
\hline Solanum mauritianum Scop. & 1 & - & - \\
\hline Solanum sanctaecatharinae Dunal & 1 & - & 7660 \\
\hline \multicolumn{4}{|l|}{ STYRACACEAE } \\
\hline Styrax leprosus Hook. \& Arn. & 1 & 2 & 7672 \\
\hline \multicolumn{4}{|l|}{ SYMPLOCACEAE } \\
\hline Symplocos tetrandra (Mart.) Miq. & - & 1 & - \\
\hline Symplocos uniflora (Pohl) Benth. & 6 & 3 & 7713 \\
\hline \multicolumn{4}{|l|}{ VERBENACEAE } \\
\hline Duranta vestita Cham. & 16 & 17 & 7656 \\
\hline \multicolumn{4}{|l|}{ WINTERACEAE } \\
\hline Drimys brasiliensis Miers & 17 & 25 & 7662 \\
\hline Total & 741 & 773 & \\
\hline
\end{tabular}

nc: Número de coleta das espécies incorporadas no Hérbario LUSC, da Universidade do Estado de Santa Catarina.

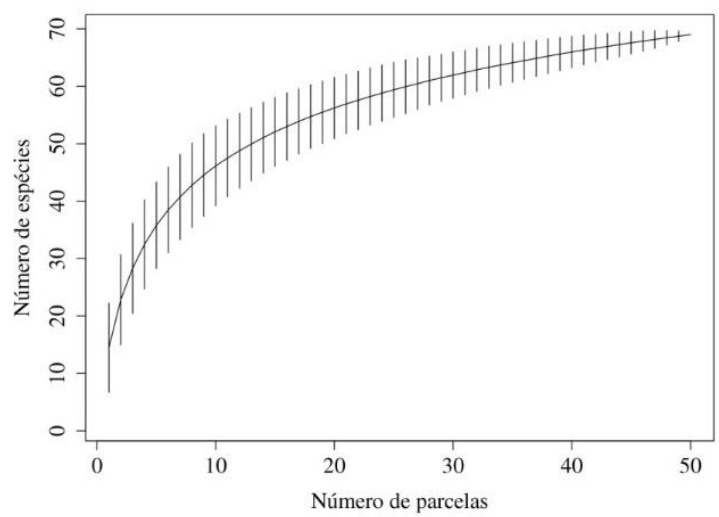

Figura 1. Curva de acumulação de espécies do fragmento de Floresta Ombrófila Mista estudado em Lages, SC.

Figure 1. Species accumulation curve of the studied Araucaria Forest patch, in Lages, SC.

Entre as 10 espécies de maior valor de importância na comunidade, Araucaria angustifolia (Bertol.) Kuntze se destacou como a espécie de maior IVI tanto no setor "borda" (Figura 2A) como no "interior" (Figura 2B). Isso se deu em função de sua elevada área basal, de 4,59 m²/ha no setor "borda" e de 4,50 $\mathrm{m}^{2} / \mathrm{ha}$ no "interior", elevada densidade (166 ind./ha no setor borda e 138 ind./ha no interior) e alta frequência (96\% na "borda" e 84\% no "interior"). Lithraea brasiliensis Marchand foi a segunda espécie de maior importância no setor "borda" e a terceira de maior importância no "interior". No "interior", Jacaranda puberula Cham. foi quem ocupou a segunda colocação de importância, porém não esteve entre as 10 espécies de maior IVI na "borda". A elevada importância nos dois setores dessas duas espécies, assim como em diferentes fragmentos de FOM (RONDON-NETO et al., 2002; CURCIO et al., 2006; CENCI et al., 2013; FERREIRA et al., 2013), sugere que essas espécies apresentam elevada plasticidade ecológica, com capacidade de desenvolvimento em diversas condições ambientais. Considerando o

FLORESTA, Curitiba, PR, v. 45, n. 2, p. 421 - 430, abr. / jun. 2015.

Pscheidt, F. et al.

ISSN eletrônico 1982-4688 / ISSN impresso 0015-3826

DOI: $10.5380 /$ rf.v45i2.34180 
histórico tradicional de perturbação das áreas com floresta com araucárias no Sul do Brasil, caracterizado pelo intenso corte seletivo de madeiras no passado, a dominância de ambas as espécies também poderia sugerir um processo de homogeneização biológica nessas áreas, como observado em fragmentos de Floresta Atlântica no Nordeste brasileiro (LÔBO et al., 2011).

De forma geral, percebe-se uma mudança florístico-estrutural entre "borda" e "interior". Apesar de Araucaria angustifolia e Lithraea brasiliensis terem apresentado maior importância em ambos os setores, houve mudança estrutural nas demais posições de IVI, com altos valores de IVI (acima de 4\%) para Myrsine lorentziana (Mez) Arechav. e Vernonanthura discolor (Spreng.) H.Rob. na "borda", espécies de início de sucessão não encontradas entre as 10 espécies de maior IVI no "interior". O mesmo ocorreu com Jacaranda puberula, Dicksonia sellowiana Hook. e Lamanonia ternata Vell., que ocorreram com alto IVI (acima de 4\%) somente no "interior".
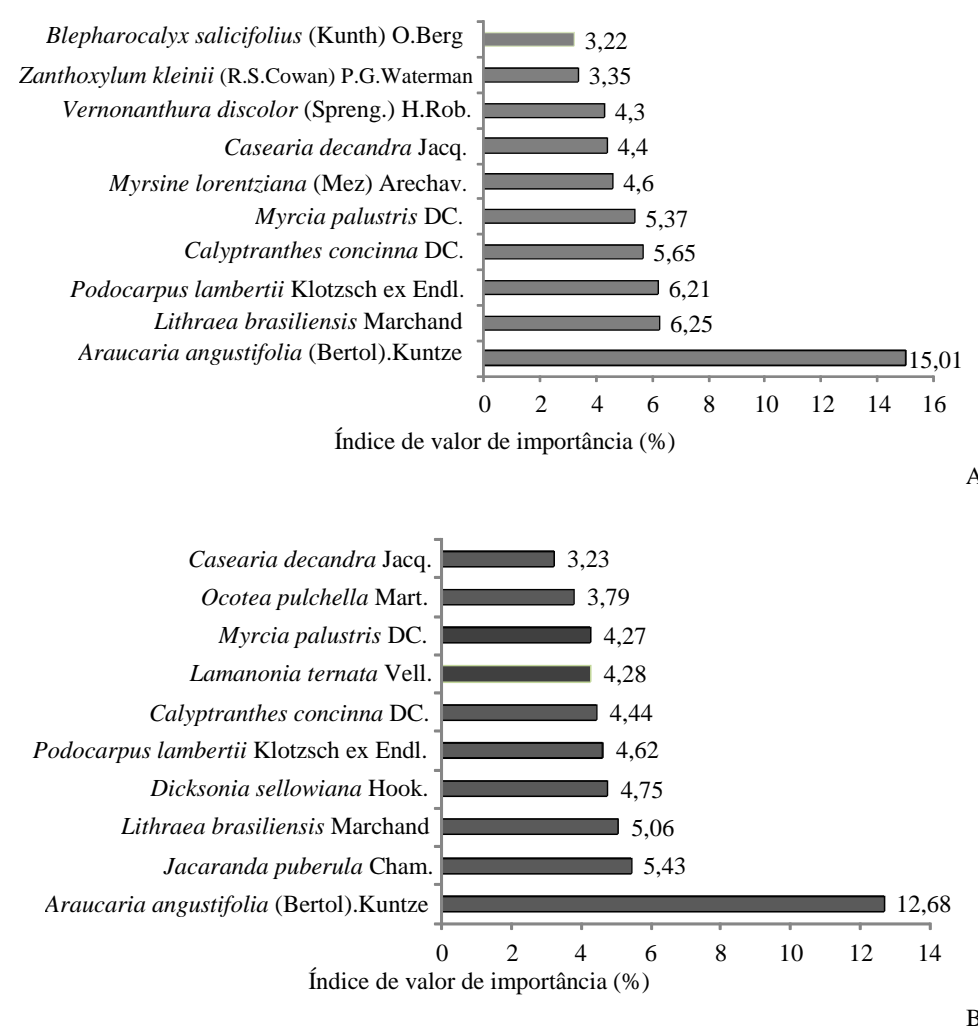

Figura 2. Índice de valor de importância das 10 espécies de maior importância no setor "borda" (A) e no setor "interior" (B) no fragmento de Floresta Ombrófila Mista estudado em Lages, SC.

Figure 2. Importance value index for the 10 most important species in 'edge' (A) and 'interior' (B) sectors, in the studied Araucaria Forest patch in Lages, SC.

As diferenças das participações relativas nas principais populações refletem a existência de variações florístico-estruturais entre os setores, como demonstrada pela ordenação das parcelas (Figura 3 ) e confirmada pela NPMANOVA $(p=0,022)$. Enquanto as parcelas de "borda" ocorreram de forma mais coesa na ordenação, as de "interior" ocorreram mais espaçadas, sugerindo maior heterogeneidade na sua organização. Apesar das variações encontradas, os autovalores dos eixos 1 e 2, respectivamente de 0,2764 e 0,1617 , são considerados baixos, indicando um gradiente curto, com baixa substituição florística entre os setores, de forma que as variações são predominantemente na abundância das espécies (TER BRAAK, 1995). Fontoura et al. (2006) também observaram mudanças vegetacionais ao longo de gradientes de borda em um fragmento de Floresta Ombrófila Mista, indicando que nessa fitofisionomia a borda representa uma importante fonte de heterogeneidade florístico-estrutural, em resposta à interação de 
diferentes fatores, como os abióticos (luminosidade, umidade e vento) e bióticos (ação de predadores) (MURCIA, 1995; BALDISSERA; GANADE, 2005).

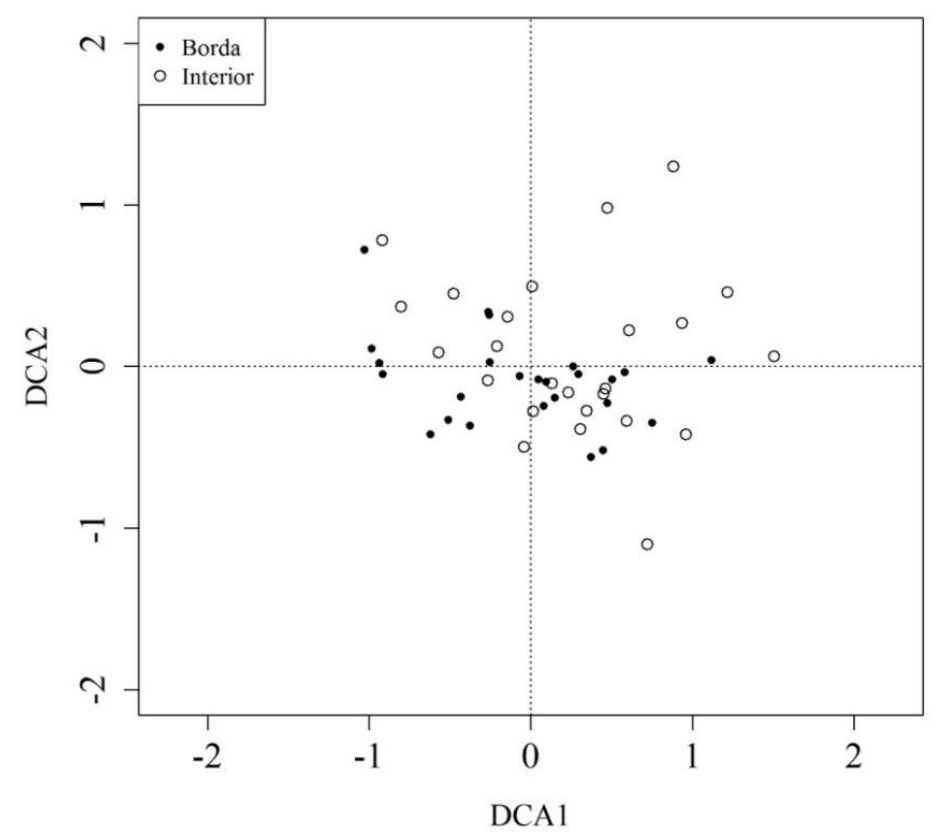

Figura 3. Análise de correspondência retificada (DCA) com parcelas do setor "borda" e "interior" do fragmento estudado de Floresta Ombrófila Mista em Lages, SC.

Figure 3. Detrended correspondence analysis (DCA) with plots of 'edge' and 'interior' sectors within the studied Araucaria Forest patch, in Lages, SC.

Apesar das variações florístico-estruturais, não foi possível verificar diferenças relativas a abundância, área basal, altura média, riqueza, diversidade e equabilidade ( $<<0,10)$ (Tabela 2), bem como quanto à distribuição de indivíduos em classes de tamanhos (Figura 4) entre os setores. Para as classes de diâmetro, a frequência de indivíduos foi maior nas classes de menor tamanho, seguindo a distribuição em J-invertido (exponencial negativa) (Figura 4A). Para altura, observou-se maior abundância de indivíduos nas classes centrais do histograma (Figura 4B). A similaridade estrutural entre setores representa um padrão diferente do observado em outros fragmentos no domínio da Floresta Atlântica (OLIVEIRAFILHO et al., 2004; FONTOURA et al., 2006; CARVALHO et al., 2007), podendo ser reflexo de um histórico de perturbação pretérito, como ocorreu na região, em função do corte de espécies madeireiras no passado. Isso reforça a ideia de que o efeito de borda sobre comunidades de espécies arbóreas não deve ser generalizado, em função de sua natureza complexa (LAURANCE et al., 2007).

Tabela 2. Densidade, área basal, altura média, riqueza, diversidade (H') e equabilidade (J) nos setores "borda" e "interior" de um fragmento de Floresta Ombrófila Mista em Lages, SC ( $p$ : significância do teste $\mathrm{t}$ ).

Table 2. Density, basal area, mean height, richness, diversity (H') and evenness (J) in 'edge' and 'interior' sectors in the studied Araucaria Forest patch in Lages, SC ( $p$ : $\mathrm{t}$ test significance).

\begin{tabular}{lccc}
\hline & Borda & Interior & $\boldsymbol{p}$ \\
\hline Densidade (ind./ha) & 1482 & 1546 & 0,7376 \\
Área basal (m²/ha) & 34,06 & 38,18 & 0,2001 \\
Altura média (m) & 8,15 & 8,33 & 0,6636 \\
Riqueza (n.esp.) & 56 & 57 & 0,3624 \\
H' (nats/indiv.) & 3,35 & 3,51 & 0,3038 \\
J' & 0,83 & 0,86 & 0,7973 \\
\hline
\end{tabular}

FLORESTA, Curitiba, PR, v. 45, n. 2, p. 421 - 430, abr. / jun. 2015.

Pscheidt, F. et al.

ISSN eletrônico 1982-4688 / ISSN impresso 0015-3826 

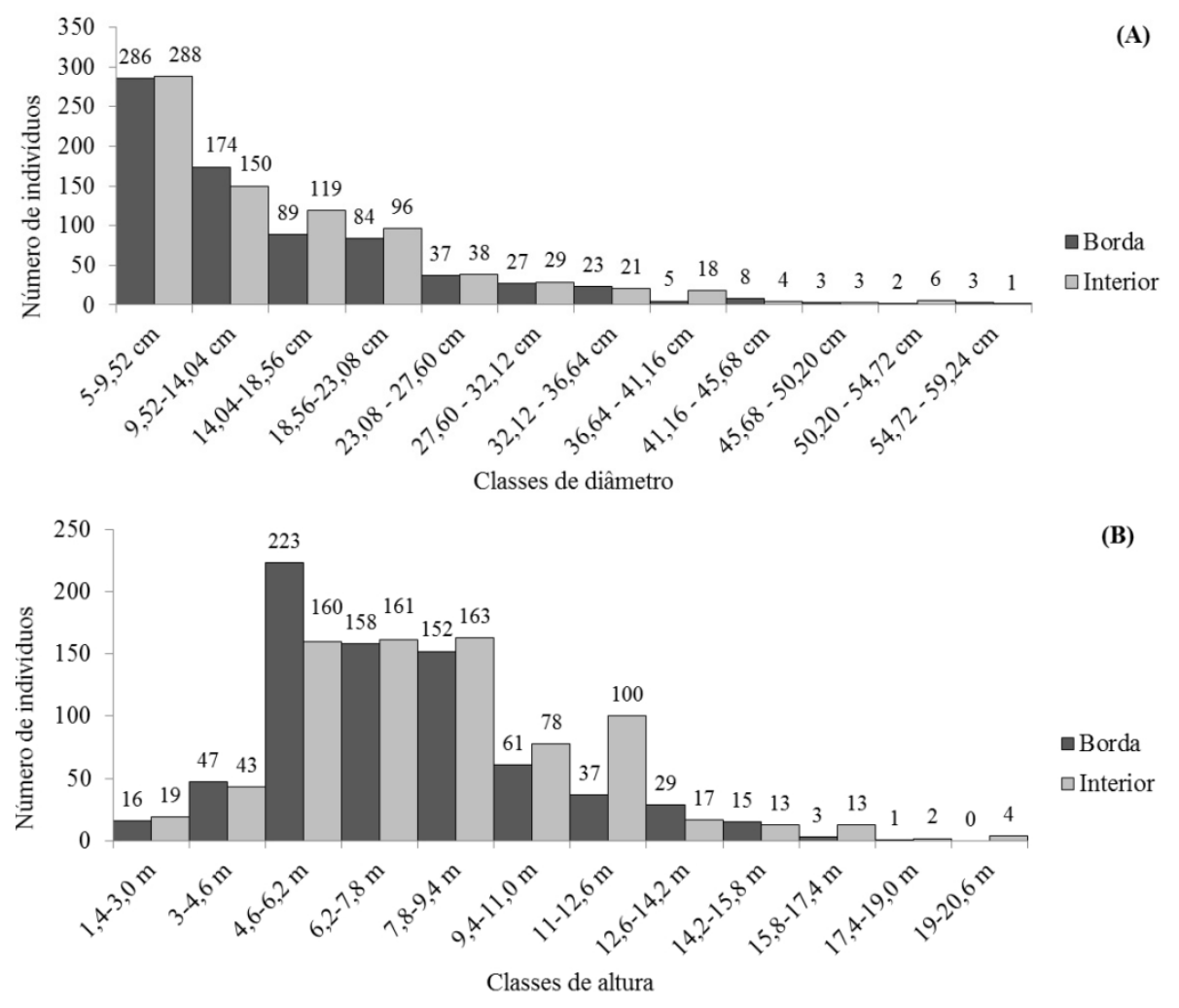

Figura 4. Estrutura diamétrica (A) e hipsométrica (B) dos indivíduos amostrados nos setores "borda" e "interior" do fragmento estudado de Floresta Ombrófila Mista em Lages, SC.

Figure 4. Diametric (A) and hypsometric (B) structure of individuals sampled in 'edge' and 'interior' sectors in the studied Araucaria Forest patch in Lages, SC.

\section{CONCLUSÃO}

Ambos os setores, "borda" e "interior", apresentaram Araucaria angustifolia e Lithraea brasiliensis como espécies de maior importância relativa e Myrtaceae como a família de maior riqueza, não demonstrando diferenças significativas em algumas variáveis mensuradas (densidade, área basal, altura média, riqueza, diversidade, equabilidade e frequência de indivíduos em classes de diâmetro e altura). No entanto, foi possível detectar substituições entre as espécies de maior VI entre os setores, que resultou em variações na organização da comunidade em função de alterações florístico-estruturais. Dessa forma, apesar de não permitir generalizações, o presente estudo representa uma contribuição para o entendimento da ecologia de comunidades de espécies arbóreas em fragmentos de Floresta Ombrófila Mista, pois demonstra que, no gradiente de borda avaliado $(0-100 \mathrm{~m})$, parte da variação florísticoestrutural na área de estudo foi determinada pela presença de bordas.

\section{AGRADECIMENTOS}

Ao CNPq, pelo financiamento do projeto, por meio do Edital MCT/CNPq 14/2010, processo 475095/2010-3, e à FUMDES/SC, pela bolsa concedida ao primeiro autor.

\section{REFERENCIAS}

ANDERSON, M. J. A new method for non-parametric multivariate analysis of variance. Austral Ecology, v. 26, n. 1, p. $23-42,2001$. 
ANGIOSPERM PHYLOGENY GROUP. An update of the Angiosperm Phylogeny Group classification for the orders and families of flowering plants: APG III. Botanical Journal of the Linnean Society, v. 161, n. 2, p. 105 - 121, 2009.

BALDISSERA, R.; GANADE, G. Predação de sementes ao longo de uma borda de Floresta Ombrófila Mista e pastagem. Acta Botanica Brasilica, v. 19, n. 1, p. 161 - 165, 2005.

BAPTISTA, S. R.; RUDEL, T. K. A re-emerging Atlantic Forest? Urbanization, industrialization and the forest transition in Santa Catarina, southern Brazil. Environmental Conservation, v. 33, n. 3, p. 195 - 202, 2006.

CARVALHO, W. A. C.; OLIVEIRA FILHO, A. T.; FONTES, M. A. L.; CURI, N. Variação espacial da estrutura da comunidade arbórea de um fragmento de floresta semidecídua em Piedade do Rio Grande, MG, Brasil. Revista Brasileira de Botânica, v. 30, n. 2, p. 315 - 335, 2007.

CENCI, B. T.; DORNELES, L. T.; SIMIONI, E. L.; FRIZON, S.; TRAVI, V. H. Composição da flora arbórea e arborescente no Jardim Botânico de Bento Gonçalves, Rio Grande do Sul, Brasil. Revista Árvore, v. 37, n. 1, p. 137 - 149, 2013.

CURCIO, G. R.; BONNET, A.; PESTANA, D.; SOUZA, L.; SOCHER, L. G.; GALVÃO, F.; RODERJAN, C. V. Compartimentação topossequencial e caracterização fitossociológica de um capão de Floresta Ombrófila Mista. Floresta, v. 36, n. 3, p. 361 - 369, 2006.

ESKUCHE, U. El bosque de Araucaria con Podocarpus y los campos de Bom Jardim da Serra, Santa Catarina (Brasil meridional). Boletín de la Sociedad Argentina de Botánica, v. 42, n. 3 - 4, p. 295 - 308, 2007.

FELFILI, J. M.; REZENDE, R. P. Conceitos e métodos em fitossociologia. Brasília: Universidade de Brasília, 2003. 68 p.

FERREIRA, P. I.; GOMES, J. P.; BATISTA, F.; BERNARDI, A. P.; COSTA, N. C.; BORTOLUZZI, R. C.; MANTOVANI, A. Espécies potenciais para recuperação de áreas de preservação permanente no Planalto Catarinense. Floresta e Ambiente, v. 20, n. 2, p. 73 - 182, 2013.

FONTOURA, S. B.; GANADE, G.; LAROCCA, J. Changes in plant community diversity and composition across edge between Araucaria Forest and pasture in South Brazil. Brazilian Journal of Botany, v. 29, n. 1, p. 79 - 91, 2006.

FORMAN, R. T. T.; GRODON, R. Landscape Ecology. New York: John Wiley e Sons Inc., 1986. 712 p.

FORMENTO, S.; SCHORN, L. A.; RAMOS, R. A. B. Dinâmica estrutural arbórea de uma Floresta Ombrófila Mista em Campo Belo do Sul, SC. Cerne, v. 10, n. 2, p. 196 - 212, 2004.

HIGUCHI, P.; OLIVEIRA FILHO, A. T.; BEBBER, D. P.; BROWN, N. D.; SILVA, A. C.; MACHADO, E. L. M. Spatio-temporal patterns off tree community dynamics in a tropical forest fragment in south-east Brazil. Plant Ecology, v. 199, n. 1, p. 125 - 135, 2008.

HIGUCHI, P.; SILVA, A. C.; FERREIRA, T. S.; SOUZA, S. T.; GOMES, J. P.; SILVA, K. M.; SANTOS, K. F.; LINKE, C.; PAULINO, P. S. Influência de variáveis ambientais sobre o padrão estrutural e florístico do componente arbóreo, em um fragmento de Floresta Ombrófila Mista Montana em Lages, SC. Ciência Florestal, v. 22, n. 1, p. 79 - 90, 2012.

HIGUCHI, P.; SILVA, A. C.; ALMEIDA, J. A.; BORTOLUZZI, R. L. C.; MANTOVANI, A.; FERREIRA, T. S.; SOUZA, S. T.; GOMES, J. P.; SILVA, K. M. Florística e estrutura do componente arbóreo e análise ambiental de um fragmento de Floresta Ombrófila Mista Alto-Montana no município de Painel, SC. Ciência Florestal, v. 23, n. 1, p. 163 - 174, 2013.

INSTITUTO BRASILEIRO DE GEOGRAFIA E ESTATÍSTICA (IBGE). Manual técnico da vegetação brasileira. Rio de Janeiro: Fundação Instituto Brasileiro de Geografia e Estatística, 2012. 271 p.

KERSTEN, R. A.; GALVÃO, F. Suficiência amostral em inventários florísticos e fitossociológicos. In: FELFILI, J. M.; EISENLOHR, P. V.; MELO, M. M. R. F.; ANDRADE, L. A.; MEIRA NETO, J. A. A. Fitossociologia no Brasil: Métodos e estudos de casos. Viçosa: Editora UFV, 2011. p. 153 - 176.

FLORESTA, Curitiba, PR, v. 45, n. 2, p. 421 - 430, abr. / jun. 2015. 
KLAUBERG, C.; PALUDO, G. F.; BORTOLUZZI, R. L. C.; MANTOVANI, A. Florística e estrutura de um fragmento de Floresta Ombrófila Mista no Planalto Catarinense. Biotemas, v. 23, n. 1, p. 35 - 47, 2010.

LAURANCE, W. F.; NASCIMENTO, H. E. M.; LAURANCE, S. G.; ANDRADE, A.; EWERS, R. M.; HARMS, K. E.; LUIZÃO, R. C. C.; RIBEIRO, J. E. Habitat fragmentation, variable edge effects, and the landscape-divergence hypothesis. PLoS ONE, v. 2, n. 10, p. 1 - 8, 2007.

LÔBO, D.; LEÃO, T.; MELO, F. P. L.; SANTOS, A. M. M.; TABARELLI, M. Forest fragmentation drives Atlantic forest of northeastern Brazil to biotic homogenization. Diversity and Distribution, v. 17, n. 2, p. 287 - 296, 2011.

MATTEUCCI, S. D.; COLMA, A. Metodologia para el estudio de la vegetacion. Washington: The General Secretarial of the Organization of American States, 1982. 167 p. (Série Biologia Monografia, 22).

MUELLER-DOMBOIS; D.; ELLENBERG, H. Aims and methods of vegetation ecology. New York: Wiley, 1974. $547 \mathrm{p}$.

MURCIA, C. Edge effects in fragmented forest: implications for conservation. Trends in Ecology \& Evolution, v. 10, n. 2, p. 58 - 62, 1995.

NASCIMENTO, A. R. T.; RAMOS, P. H. X.; DALMASO, C. A. Estrutura e classificação de um remanescente de floresta ripária no município de Lages, SC. Ciência Florestal, v. 21, n. 2, p. 209 - 218, 2011.

OKSANEN, J.; BLANCHET, F. G.; KINDT, R.; LEGENDRE, P.; MINCHIN, P. R.; O’HARA, R. B.; SIMPSON, G. L.; SOLYMOS, P.; STEVENS, M. H.; WAGNER, H. Vegan: Community Ecology Package. R package version 1.17-2. Disponível em: 〈http://CRAN.R-project.org/package=vegan>. Acesso em: 15/05/2013.

OLIVEIRA-FILHO, A. T.; CARVALHO, D. A.; FONTES, M. A. L.; VAN DEN BERG, E.; CURI, N.; CARVALHO, W. A. C. Variações estruturais do compartimento arbóreo de uma Floresta Semidecídua Alto-Montana na chapada das Perdizes, Carrancas, MG. Revista Brasileira de Botânica, v. 27, n. 2, p. 291 - 309, 2004.

R DEVELOPMENT CORE TEAM. R: A language and environment for statistical computing. $R$ Foundation for Statistical Computing. Disponível em: <http://www.R-project.org>. Acesso em: $15 / 05 / 2013$.

RIBEIRO, M. C.; METZGER, J. P.; MARTENSEN, A. C.; PONZONI, F. J.; HIROTA, M. M. The Brazilian Atlantic Forest: How much is left, and how is the remaining forest distributed? Implications for conservation. Biological Conservation, v. 142, n. 6, p. 1141 - 1153, 2009.

RONDON-NETO, R. M.; WATZLAWICK, L. F.; CALDEIRA, M. V. W.; SCHOENINGER, E. R. Análise florística e estrutural de um fragmento de Floresta Ombrófila Mista Montana, situado em Criúva, RS, Brasil. Ciência Florestal, v. 12, n. 1, p. 29 - 37, 2002.

SILVA, A. C.; HIGUCHI, P.; AGUIAR, M. D.; NEGRINI, M.; FERT NETO, J.; HESS, A. F. Relações florísticas e fitossociologia de uma Floresta Ombrófila Mista Montana secundária em Lages, Santa Catarina. Ciência Florestal, v. 22, n. 1, p. 193 - 206, 2012.

TER BRAAK, C. J. F. Ordination. In: JONGMAN, R. H. G.; TER BRAAK, C. J. F.; VAN TONGEREN, O. F. R. Data analysis in community and landscape ecology. Cambridge: Cambridge University Press, 1995. p. $91-173.212$ p.

VIANA, V. M.; PINHEIRO, L. A. F. V. Conservação da biodiversidade em fragmentos florestais. Série Técnica IPEF, v. 12, n. 32, p. 25 - 42, 1998.

YOUNG, A.; MITCHEL, N. Microclimate and vegetation edge effects in a fragmented podocarpbroadleaf forest in New Zeland. Biological Conservation, v. 67, n. 1, p. 63 - 72, 1994. 